\title{
Early Predictors of Suboptimal Response to CML Therapy Could Help in Determining Treatment Strategy
}

\author{
Mohammed M.A. EID ${ }^{1}$, Marwa M.A. Abd El HAFEEZ ${ }^{1}$, Hend N. ELLITHY ${ }^{2}$, \\ Heba M.Z. ELABDIN³ , Ghada M. EZZAT ${ }^{1}$ \\ ${ }^{1}$ Fayoum University, Faculty of Medicine, Department of Clinical Pathology \\ ${ }^{2}$ Cairo University, Faculty of Medicine, Department of Clinical Hematology \\ ${ }^{3}$ Fayoum University, Faculty of Medicine, Department of Internal Medicine, Fayoum City, EGYPT
}

\begin{abstract}
Chronic myeloid leukemia (CML) respond dramatically to molecular target therapy; imatinib (IM), a first generation tyrosine kinase inhibitor (TKIs). Quantitation of cytokines like Interleukin-6, Interleukin-7 and Transforming growth factor- $\alpha$ plasma levels before IM therapy, could assess early molecular response (EMR) to IM and predict imatinib failure. A case-control study of $30 \mathrm{CML}$ patients and 30 controls. Levels of IL-6, IL-7 and TGF- $\alpha$ were assayed by ELISA (R\&D systems, USA) for both controls and patients. The patients' BCR-ABL1 transcript was assayed by real time-quantitative polymerase chain reaction, using ipsogen® BCR-ABL1 Mbcr Kit on the Rotor-Gene Q MDx (Qiagen, USA). Cytokines and BCR-ABL1 levels were done both before therapy and at 3 months follow up. Three months following IM therapy, the patients were divided into improved $(n=27)$ and non-improved $(n=3)$ groups; based on the establishment of EMR. Plasma levels of IL-7, IL-6 and TGF- $\alpha$ were significantly higher in CML patients $(p<0.05)$. Cytokines plasma levels dropped significantly after IM therapy $(p<0.05)$. Correlation studies revealed a strong positive correlation between pretreatment levels of both IL- 6 and TGF- $\alpha$ and posttreatment levels of BCR-ABL transcript ( $r=0.89$ and 0.84 , respectively). IL-7 showed a poor correlation with posttreatment levels of BCR-ABL transcript ( $r=0.32$ ). Our study revealed a possible role of IL-6, IL-7 and TGF- $\alpha$ as mediators of CML. The initial high levels of IL-6 and TGF- $\alpha$ was associated with the failure of achieving EMR. The initial high levels of IL-7 in CML patients appears to facilitate the disease process.
\end{abstract}

Keywords: Chronic myeloid leukemia, Imatinib, Early molecular response

\section{INTRODUCTION}

Chronic myeloid leukaemia (CML) is probably one of the most comprehensively studied human malignancies. CML was the first human cancer that responds to molecular target therapy, imatinib, a member of tyrosine kinase inhibitors (TKIs). ${ }^{1}$ Despite the excellent efficacy of imatinib, a subset of patients does not respond properly to $\mathrm{it}^{2}$, and are deemed to have resistance to the drug. Several mechanisms can play a role in the resistance to TKIs. ${ }^{3}$ The resistance to first generation TKIs in CML patient may indicate that stem cells are not dependent on BCR-ABL1 kinase for their survival, suggesting that kinase-independent mechanisms contribute to their survival. ${ }^{4}$

Tumor microenvironment plays a critical role in tumor initiation, progression and response to the treatment. Angiogenesis, a key rate-limiting step in the growth and dissemination of malignant tumors, is regulated by cytokines. Interleukin-6 (IL-6) is one of important cytokines involved in the vascularization of tumors. ${ }^{5}$ IL- 6 has been demonstrated to directly stimulate proliferation of tumor cells and promote angiogenesis. 
Several reports have found that IL-6 levels correlate with disease progression and inversely correlate with response to treatment and survival. ${ }^{6-8}$

In myeloproliferative neoplasms serum levels of interleukin (ILs)-1a, IL-1b, IL-2 and IL-6 revealed interesting results as Cytokines profiling has proven valuable to identify prognostic factor in myelofibrosis and myelodysplastic syndromes, but similar comprehensive studies are lacking to date in CML. ${ }^{9}$ Soluble factors produced in the bone marrow, such as growth factors like transforming (tumor) growth factor- $\alpha$ (TGF- $\alpha$ ) mediate homing, survival and proliferation of tumor cells. ${ }^{10}$ IL-7 plays a significant role in hematological malignancies. Relevantly, Zhang and his colleagues in 2016 proved that high IL-7 levels in bone marrow microenvironment is associated with resistance to imatinib therapy. ${ }^{11}$ These data prompted the fact that IL-7 could be a relevant marker in predicting response to imatinib therapy.

Our current study aims at quantifying serum levels of IL-7, IL- 6 and TGF- $\alpha$ at time of CML diagnosis before beginning imatnib therapy, and assessing their possible usage as biomarkers for predicting subsequent early molecular response (EMR), failure to this therapy for proper strategic therapy fitting.

\section{PATIENTS and METHODS}

A case-control study was conducted on $30 \mathrm{CML}$ patients who were evaluable for molecular analysis at diagnosis and at 3 months follow up of IM therapy. The patients presented to the Outpatient Clinics of the Hematology Department at Kasr AlAiny Hospital, Cairo University from May 2015 to March 2016. Also 30 adult healthy controls, matched in age and sex with the selected patients were enrolled. This study was approved by Fayoum Faculty of Medicine Research Ethics Committee which is a member of the Egyptian Network and Research Ethics Committees (ENREC). Informed consent was obtained from all subjects. Inclusion criteria was newly diagnosed adult patients with morphologic and molecular/cytogentic evidence of CML in all clinical stages as defined by the WHO 2016 criteria. $^{12}$ Exclusion of pregnant females as well as patients with significant renal, hepatic and cardiac impairment was considered. All patients were subjected to full medical history taking and complete clinical examination for the presence of hepatomegaly, splenomegaly. Data collection from patients' files included their initial laboratory investigations (complete blood picture, leukocyte alkaline phosphatase (LAP) score, kidney and liver function tests and bone marrow examination) and abdominal ultrasound results. Sokal ${ }^{13}$, Hasford ${ }^{14}$ and EUTOS $^{15}$ prognostic scores were calculated for all patients at presentation. Sterile venous blood was collected and divided in serum separator tubes and EDTA vacutainers. Serum was separated and divided into aliquots and stored at $-80^{\circ} \mathrm{C}$ until used for cytokines assay. EDTA samples were used for molecular assay. Sample collection for both cytokines profiling and molecular analysis was done at the time of diagnosis and at 3 months after imatinib therapy for patients. IL-6, IL-7 and TGF- $\alpha$ serum levels were also done for the control group. Imatinib was given at a dose of $400 \mathrm{mg}$ orally once a day. Hydroxyurea was used before imatinib for the treatment of elevated platelets $\left(>700 \times 10^{9} / \mathrm{L}\right)$ and WBC count $\left(>50 \times 10^{9} / \mathrm{L}\right)$ for 2 weeks. Interleukin-6 (IL-6), Interleukin-7 (IL-7) and Transforming growth factor-alpha (TGF- $\alpha$ ) were quantitated by enzyme-linked immunosorbent assay (ELISA) employing the sandwich technique (R\&D systems, USA). All readings were measured using Elx800 ELISA reader (BioTek, USA). Quantitative real-time PCR assay for BCR-ABL1 transcript was done using ipsogen BCR-ABL1 Mbcr Kit on the Rotor-Gene Q Mdx (Qiagen, USA). The definition of hematologic, and molecular responses was defined according to WHO $2016 .{ }^{12}$

\section{Statistical Analysis}

Data was collected and coded to facilitate data manipulation and double entered into Microsoft Access and all analyses were performed using the statistical package for the social sciences (SPSS software 17; SPSS Inc., Chicago, USA). For quantitative parametric data, independent student t-Test was used to compare two independent groups, while paired t-test in comparing two dependent groups. For quantitative non-parametric data, Mann-whitney test was used to compare two 
International Journal of Hematology and Oncology

\begin{tabular}{|c|c|c|c|c|c|}
\hline Parameter & $\begin{array}{l}\text { Controls } \\
(n=30)\end{array}$ & $\begin{array}{l}\text { CML (pre- } \\
\text { treatment) } \\
(n=30)\end{array}$ & $\begin{array}{l}\text { CML (post- } \\
\text { treatment) } \\
(n=30)\end{array}$ & $\begin{array}{l}\text { p-value } \\
\text { control vs } \\
\text { pre-treatment }\end{array}$ & $\begin{array}{l}\text { p-value } \\
\text { pre- vs } \\
\text { post-treatment }\end{array}$ \\
\hline Age & $48.4 \pm 7.2$ & $51.3 \pm 17.4$ & NS & NA & \\
\hline Blast \% & 0 & $3.5 \pm 6.9$ & $0.26 \pm 0.9$ & $<0.001$ & $<0.001$ \\
\hline Basophils \% & $1.3 \pm 0.11$ & $5.8 \pm 8.3$ & $1.6 \pm 1$ & $<0.001$ & $<0.001$ \\
\hline Eosinophils \% & $4.1 \pm 0.3$ & $1.9 \pm 1.1$ & $3.2 \pm 1.6$ & NS & NS \\
\hline Platelets $\left(10^{3} / \mu \mathrm{L}\right)$ & $249 \pm 97$ & $288.2 \pm 184.5$ & $232.1 \pm 96$ & NS & NS \\
\hline Spleen size (cm) & Not palpable & $17.2 \pm 6.2$ & $0.73 \pm 2.3$ & $<0.001$ & $<0.001$ \\
\hline IL-7 (pg/mL) & $10.9 \pm 3.8$ & $12.6 \pm 4.06$ & $4.7 \pm 3.2$ & 0.053 & $<0.001$ \\
\hline IL-6 (pg/mL) & $1.9 \pm 1.8$ & $38.2 \pm 63.3$ & $13.9 \pm 23.6$ & $<0.001$ & $<0.001$ \\
\hline TGF- $\alpha(p g / m L)$ & $13.8 \pm 4.3$ & $72.4 \pm 58.1$ & $29.6 \pm 13.9$ & $<0.001$ & $<0.001$ \\
\hline BCR-ABL1\% & NA & $114.2 \pm 124.7$ & $15.9 \pm 39.9$ & $<0.001$ & $<0.001$ \\
\hline
\end{tabular}

independent groups, while Wilcoxon test was used to compare two groups of dependant data. For qualitative data, chi square test was used to compare two or more groups, while bivariate Pearson correlation test was used to examine association between variables. $\mathrm{P}$ value $\leq 0.05$ was considered the cut-off value for significance.

\section{RESULTS}

\section{Patients Expressed Distinctive Cytokine Profiles}

This prospective study included thirty newly diagnosed adult patients who were evaluable for molecular analysis at diagnosis and at 3 moths of IM therapy. Sixty percent of evaluable patients were males and $40 \%$ were females. We further classified our CML patients into 3 categories, chronic phase CML (CP-CML), acute phase CML (AP-CML), and blast phase (BP-CML), representing the progressive phases of CML.

Analysis of the patients' results revealed that IL-7, IL- 6 and TGF- $\alpha$ are significantly increased in CML patients compared to controls (Table 1). These high levels dropped significantly after 3 months of IM therapy. Similarly, all other parameters, with exception of eosinophils and platelets, dropped significantly after 3 months of IM. These data confirmed the notion that cytokines, particularly IL-7, IL- 6 and TGF- $\alpha$ have a role in disease progression and leukemic cells proliferation.

\section{IL-7, IL-6 and TGF- $\alpha$ Levels Correlate with CML Disease Activity}

Table (2) demonstrates the levels of IL-7, IL-6, TGF- $\alpha$ and BCR-ABL1\% in different CML categories. The 3 cytokines, IL-7, IL- 6 and TGF- $\alpha$ expressed higher levels in CP-CML compared to controls, the levels are more increased in AP-CML and further in BP-CML, with exception of IL-7 which is not further increased in BP-CML (Table $2)$. This data highly suggests that the plasma levels of these cytokines correlate with CML disease activity. Confirming this notion, the correlation studies revealed a strong correlation between IL-6 and BCR-ABL1, blast number and the Sokal and Hasford prognostic scores. A poor correlation was found between IL- 6 and EUTOS score and basophil count (Table 3). A similar trend was found for TGF- $\alpha$ (Table 3). However, IL-7 exhibited a poor correlation with all markers, as well as poor correlation with IL-6 and TGF- $\alpha$ themselves (Table 3).

\section{Altered Cytokines Profiles Between Improved and non-Improved CML Cases}

Categorization of the cases according to their response to 3 months of IM therapy revealed that $\sim 90 \%(n=27)$ of cases achieved complete hematological response (CHR), compared to $10 \%(\mathrm{n}=$ 3 ) who failed to reach the same response. We identified the first group as improved, and the second group as non-improved. Comparing the prognostic scores between 2 groups revealed that the scores 
International Journal of Hematology and Oncology

Table 2. IL-7, IL-6, TGF- $\alpha$ and BCR-ABL concentrations among CML stages

\begin{tabular}{|llllll|}
\hline Parameter & Controls $(\mathbf{n}=\mathbf{3 0})$ & Chronic $(\mathbf{n}=\mathbf{2 7})$ & Accelerated $(\mathbf{n}=\mathbf{2})$ & Blast Crisis $(\mathbf{n}=\mathbf{1})$ & Total $(\mathbf{n}=\mathbf{3 0})$ \\
\hline IL-7 $(\mathrm{pg} / \mathrm{mL})$ & $10.9 \pm 3.8$ & $12.33 \pm 4.16$ & $15.8 \pm 1.98$ & 13.6 & $12.6 \pm 4.06$ \\
IL-6 $(\mathrm{pg} / \mathrm{mL})$ & $1.9 \pm 1.8$ & $22.8 \pm 29$ & $100.32 \pm 22.6$ & 314.8 & $38.2 \pm 63.3$ \\
TGF- $\alpha(\mathrm{pg} / \mathrm{mL})$ & $13.8 \pm 4.3$ & $56.7 \pm 30.2$ & $208.7 \pm 10.3$ & 245.7 & $72.4 \pm 58.1$ \\
BCR-ABL1\% & NA & $80.2 \pm 57.7$ & $441 \pm 224.9$ & 378 & $114.2 \pm 124.7$ \\
\hline
\end{tabular}

\begin{tabular}{|llll|}
\hline \multicolumn{4}{|l|}{ Table 3. Correlations of IL-7, IL-6 (before IM therapy) } \\
\hline $\begin{array}{l}\text { Variables } \\
\text { (Before } \\
\text { treatment) }\end{array}$ & $\begin{array}{l}\text { IL-7 } \\
\text { (Before } \\
\text { treatment) } \\
\mathbf{r}\end{array}$ & $\begin{array}{l}\text { IL-6 } \\
\text { (Before } \\
\text { treatment) } \\
\mathbf{r}\end{array}$ & $\begin{array}{l}\text { TGF- } \alpha \\
\text { (Before } \\
\text { treatment) } \\
\mathbf{r}\end{array}$ \\
\hline BCR-ABL & 0.21 & 0.62 & 0.86 \\
Blasts & 0.11 & 0.88 & 0.75 \\
Basophils & 0.17 & 0.26 & 0.44 \\
Sokal score & 0.05 & 0.86 & 0.61 \\
Hasford score & 0.05 & 0.74 & 0.7 \\
EUTOS score & 0.21 & 0.33 & 0.48 \\
IL-6 & 0.16 & NA & 0.78 \\
TGF- $\alpha$ & 0.18 & 0.78 & NA \\
\hline
\end{tabular}

are significantly higher in non-improved compared to improved ones (Table 4). Next, we analyzed cytokine profiles in both groups before and after IM therapy.

Based on ROC curves, the optimal cut-off value for IL-7 is $16.37 \mathrm{pg} / \mathrm{mL}$, TGF- $\alpha$ is $49.33 \mathrm{pg} /$ $\mathrm{mL}$ and IL-6 is $8.3 \mathrm{pg} / \mathrm{mL}$ (Figure 1). IL-7, IL-6, TGF- $\alpha$ and BCR-ABL1 expressed significantly

higher levels in non-improved compared to improved cases.

Importantly, after IM treatment the levels of cytokines dropped significantly in improved group, being almost normalized to cut-off in IL- 6 and even lowered below cut-off in IL-7 and TGF- $\alpha$. The BCR-ABL1 also decreased significantly after therapy. However, in non-improved group the decrease in BCR-ABL1 and IL-6 was not significant, while a significant decrease was observed in IL-7 and TGF- $\alpha$ (Tables 4, 5).

Levels of IL-6 and TGF- $\alpha$ but not IL-7 before IM therapy correlates with BCR-ABL after therapy

As the response to IM therapy is often monitored by the level of BCR-ABL1 transcript, we studied the correlation between the pretreatment plasma levels of IL-6, TGF- $\alpha$ and IL-7 with that of BCRABL1 transcript level after IM therapy. As shown in Figure (2) pretreatment levels of both IL-6 and TGF- $\alpha$ exhibited a very strong positive correlation with post-treatment BCR-ABL1 transcript lev-

\begin{tabular}{|c|c|c|c|}
\hline Parameters & Non-improved $(n=3)$ & Improved $(n=27)$ & $\mathbf{p}$ \\
\hline \multicolumn{4}{|c|}{ Before treatment } \\
\hline BCR-ABL & $420 \pm 163$ & $80.2 \pm 57.7$ & $<0.001$ \\
\hline IL-6 & $171.8 \pm 124.9$ & $22.8 \pm 29.1$ & $<0.001$ \\
\hline TGF- $\alpha$ & $221 \pm 22.6$ & $55.8 \pm 29.9$ & $<0.001$ \\
\hline IL-7 & $15.09 \pm 1.9$ & $12.33 \pm 4.16$ & 0.049 \\
\hline Sokal score & $18.3 \pm 26$ & $1.4 \pm 0.5$ & 0.001 \\
\hline Hasford score & $2686 \pm 1329.3$ & $1342.8 \pm 451.4$ & 0.01 \\
\hline EUTOS score & $177.7 \pm 56.1$ & $99.5 \pm 64.6$ & 0.01 \\
\hline \multicolumn{4}{|c|}{ After treatment } \\
\hline BCR-ABL & $117.7 \pm 72.5$ & $4.7 \pm 6.7$ & $<0.001$ \\
\hline IL-6 & $55.3 \pm 17.8$ & $9.1 \pm 3.5$ & $<0.001$ \\
\hline TGF- $\alpha$ & $50.5 \pm 11.9$ & $27.2 \pm 2.3$ & $<0.001$ \\
\hline IL-7 & $4.53 \pm 3.27$ & $4.73 \pm 3.27$ & 0.93 \\
\hline
\end{tabular}




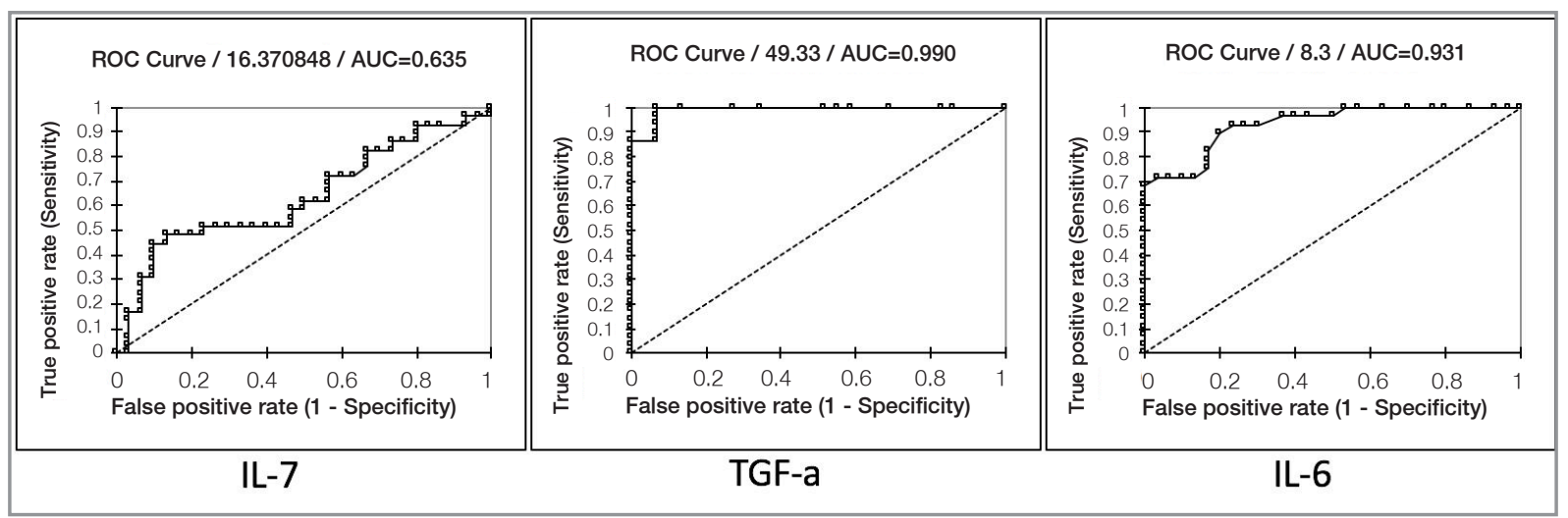

Figure 1. ROC curves for IL-7, TGF- $\alpha$, and IL-6. Based on ROC curves, the optimal cut-off value for IL-7 is $16.37 \mathrm{pg} / \mathrm{mL}$, TGF- $\alpha$ is $49.33 \mathrm{pg} / \mathrm{mL}$ and IL-6 is $8.3 \mathrm{pg} / \mathrm{mL}$

els. On the other hand, pretreatment level of IL-7 showed a poor correlation. These data mean that the high pretreatment levels of IL- 6 and TGF- $\alpha$, but not IL-7, could figure out the fate of response to IM therapy.

\section{DISCUSSION}

During the past decade, the therapy of CML has experienced unprecedented improvements of response and survival. TKIs allow a much faster reduction of BCR-ABL1 transcripts level than previously available drug therapy which is associated with a lower rate of progression and improvement of survival. ${ }^{16}$ However, still some patients continue to respond sub-optimally or become resistant and require alternative treatment with second generation TKIs or stem cell transplantation. ${ }^{17}$ So in an attempt to improve CML outcome, it was thought that early prediction of suboptimal response or failure would benefit this group of patients. ${ }^{18}$

Numerous studies proved that the lack of complete hematological response at 3 months was associ- ated with a low rate of subsequent CMR. ${ }^{19}$ According to other studies, failure of early molecular response (i.e. BCR-ABL1IS $\leq 10 \%$ at 3 months) indicates failure of achieving CCyR at 12 months and MMR at 18 months of therapy. ${ }^{20}$ Moreover, cytokine profiling of newly diagnosed CP-CML patients who subsequently received imatinib treatment revealed elevated levels of a wide range of pro-inflammatory and angiogenesis-promoting cytokines, chemokines and growth factors compared with healthy donors. Most of these normalized after tyrosine kinase inhibitor treatment while others remained high in remission samples. They identified TGF- $\alpha$ and IL- 6 as novel biomarkers with high diagnostic plasma levels strongly predictive of subsequent failure to achieve EMR and deep molecular response, as well as transformation to blast crisis and event-free survival. ${ }^{21}$

All the previous suggests that combining these simple measurements to the diagnostic workup of CP-CML patients may enable therapy to be individualized early according to the cytokine-risk profile of the patient.

\begin{tabular}{|c|c|c|c|c|c|c|c|c|c|}
\hline & \multicolumn{3}{|l|}{ Total $(n=30)$} & \multicolumn{3}{|c|}{ Improved $(n=27)$} & \multicolumn{3}{|c|}{ Non-improved $(n=3)$} \\
\hline & Before & After & $\mathbf{p}$ & Before & After & p & Before & After & p \\
\hline IL-7 (pg/mL) & $12.6 \pm 4.06$ & $4.7 \pm 3.2$ & $<0.001$ & $12.33 \pm 4.16$ & $4.73 \pm 3.27$ & $<0.001$ & $15.09 \pm 1.9$ & $4.53 \pm 3.27$ & $<0.001$ \\
\hline IL-6 (pg/mL) & $38.2 \pm 63.3$ & $13.9 \pm 23.6$ & 0.02 & $22.8 \pm 29.1$ & $9.1 \pm 3.5$ & $<0.001$ & $171.8 \pm 124.9$ & $55.3 \pm 17.8$ & 0.3 \\
\hline TGF- $\alpha(p g / m L)$ & $72.4 \pm 58.1$ & $29.6 \pm 13.9$ & $<0.001$ & $55.8 \pm 29.9$ & $27.2 \pm 2.3$ & $<0.001$ & $221 \pm 22.6$ & $50.5 \pm 11.9$ & 0.001 \\
\hline BCR-ABL1\% & $114.2 \pm 124.7$ & $15.9 \pm 39.9$ & $<0.001$ & $80.2 \pm 57.7$ & $4.7 \pm 6.7$ & $<0.001$ & $420 \pm 163$ & $117.7 \pm 72.5$ & 0.095 \\
\hline
\end{tabular}




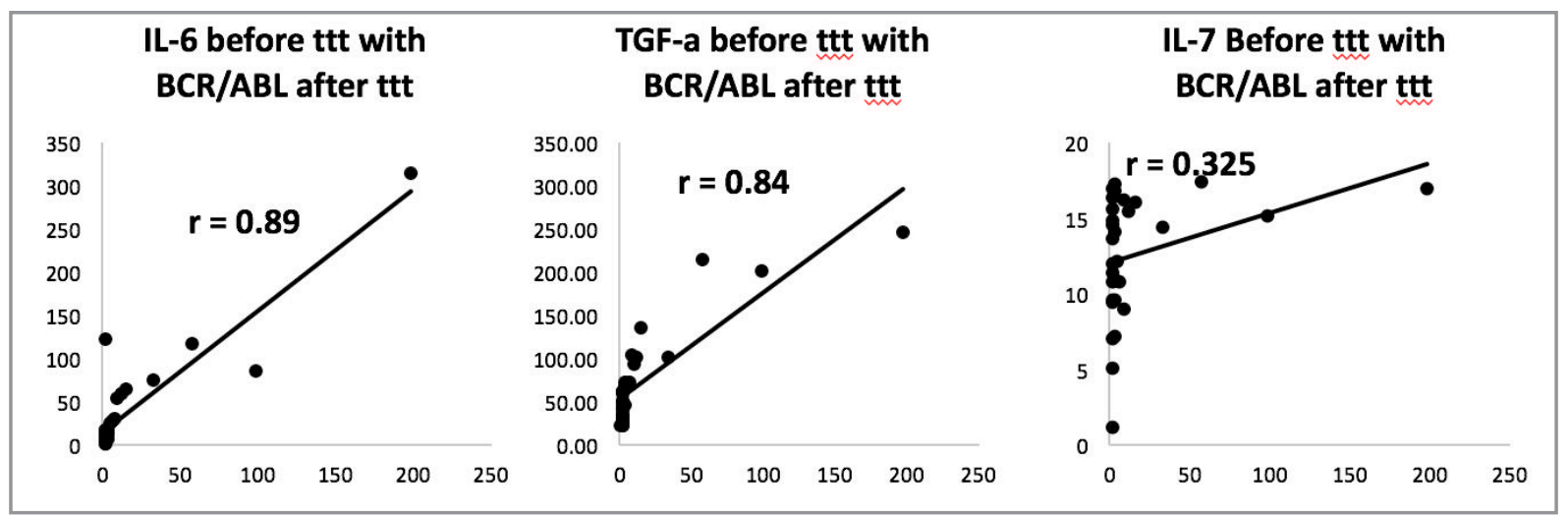

Figure 2. Correlations of IL-7, IL-6, TGF- $\alpha$ plasma levels before treatment and BCR-ABL1 after treatment. IL-6 and TGF- $\alpha$ levels showed strong correlation with BCR-ABL1 transcript levels after treatment. On the other hand, IL-7 expressed a poor correlation with BCR-ABL1 transcript levels after treatment.

Our current study aimed at measuring levels of IL7, IL-6 and TGF- $\alpha$ at the time of CML diagnosis before beginning imatnib therapy, and evaluating their possible usage as biomarkers for predicting subsequent EMR failure to this therapy for proper strategic therapy fitting.

In the present study, complete hematological response (CHR) and early molecular response (EMR) at 3 months of IM therapy was achieved by $27 / 30$ patients (90\%), which is similar to the study performed by Umemoto et al., who recorded EMR and CHR in 716 out of 822 newly diagnosed CML patients $(87.8 \%){ }^{22}$

Profiling of cytokines (Table 1), revealed that concentrations of IL-7, IL- 6 and TGF- $\alpha$ were significantly elevated in CML patients compared to controls, and these high concentrations markedly dropped after IM therapy. Considering CML stages, the cytokines' profiles revealed that levels of IL- 6 and TGF- $\alpha$ increase as the disease progresses (Table 2) and this is in compliance with the data from Nievergall and his collegues ${ }^{9}$, however, in IL-7 and BCR-ABL1, their levels increased in acute phase compared to chronic phase, but not further increased in blast phase. Collectively, these data signify that these cytokines could have a role in CML progression.

To further delineate the relation between these cytokines and disease progression, we studied the correlation of their levels with different prognostic scores (Table 3). While IL-6 and TGF- $\alpha$ both strongly correlate with Sokal and Hasford scores, and also with BCR-ABL1 and blast count, they demonstrated a poor correlation with EUTOS score. Surprisingly, levels of IL-7 expressed a poor correlation with all markers studied (Table 3 ). These data revealed that IL-6 and TGF- $\alpha$ might have a role in disease progression, however, for IL7 , it seems that it may not have an active role in CML progression but rather its initial high levels may facilitate the disease process.

It was shown previously that an association exists between IM resistance and high pretreatment levels of IL-6 and TGF- $\alpha$ (23). To analyze this notion, we divided the study subjects into improved group ( $n=27)$ and non-improved group $(n=3)$ at 3 months after IM therapy, based on the levels of BCR-ABL1 transcript as the standard method for follow up and assessing response to treatment.

Comparison between BCR-ABL1 before treatment in 2 groups (improved and non-improved) showed that there was a highly statistically significant difference with $\mathrm{p}<0.001$ (Table 4). This may indicate that there is an association between BCR-ABL1 transcript level at the diagnosis and response to IM therapy. Likewise, similar findings were found for IL-7, IL-6 and TGF- $\alpha$ levels which varied greatly between improved and non-improved groups (Table 4).

We tried to figure out if the pre-treatment levels of IL-7, IL- 6 and TGF- $\alpha$ could be a predictors of response to IM therapy. To study the relation between 
cytokines levels before treatment and response to therapy, Pearson correlation revealed a strong positive correlation between pretreatment TGF- $\alpha$ levels and BCR-ABL1 after treatment, and a similar finding was found for IL-6 (Figure 2), in agreement with data from Tefferi et al. and Reynaud et al. ${ }^{4,23}$ Surprisingly, a poor correlation was found between pretreatment IL-7 levels and BCR-ABL1 transcript $\%$. This proves the presence of a significant association between initial high IL- 6 and TGF- $\alpha$ levels and bad progression of the disease. On the other hand, although, no such correlation could be established for IL-7, however its high levels in CML patients and the dramatic reduction in its levels after IM therapy, highly suggests that it possesses some important role in disease assistance.

In summary we found positive association and correlation between high pretreatment levels of IL-6 and TGF- $\alpha$ and IM resistance. Also we found positive correlation between IL- 6 and TGF- $\alpha$ and BCRABL1 before and after treatment. Importantly, we observed a possible role for IL-7 in disease facilitation. Recently many studies were performed on basis of adding drugs beside IM which antagonize the action of IL- 6 and TGF- $\alpha$ in resistant patient to IM. These drugs have potent anti-proliferative effect, potentiate TKI effect, reducing adhesion of neoplastic cells to BM stroma and increasing apoptotic ability, on the long run there was a noticed decrease in prevalence of IM resistance and achieving optimal molecular response in previously resistant patients. ${ }^{24,25}$

Our current study could suggest that addition of drugs that antagonize IL-7 may aid in modifying disease progression and help in cases resistant to IM therapy. This could be confirmed by data from Zhang and his colleagues, who reported that high IL-7 in bone marrow mediates imatinib resistance, and targeting IL-7 pathway is a promising approach in CML therapy ${ }^{26}$ More patients need to be evaluated by multi center studies to see if evidence reported in this work is reproducible in large sample studies.

\section{CONCLUSION}

The study revealed that levels of IL-6, IL-7 and TGF- $\alpha$ levels were elevated in CML patients com- pared to controls, and dropped significantly after IM therapy; implicating their possible role as mediators of CML. The initial high levels of IL-6 and TGF- $\alpha$ was associated with the failure of achieving EMR. Thus the high levels of IL-6 and TGF- $\alpha$ at diagnosis can be used as a predictive of IM response. The high levels of IL-7 in CML patients appears to facilitate the disease process.

\section{REFERENCES}

1. Besa EC, Krishnan M. Chronic Myelogenous leukemia and clinical presentation. Br J Hematology 137: 288-296, 2013.

2. Manley PW, Cowan-Jacob SW, Buchdunger E, et al. Imatinib: a selective tyrosine kinase inhibitor. Eur J Cancer S19S27, 2011.

3. Buehler B, Marksman M, Sachet RA, et al. Hematopoietic stem cells: the paradigmatic tissue-specific stem cell. Am J Pathology 169: 338-346, 2013

4. Hamilton A, Helgason GV, Holyoake TL. Chronic myeloid leukemia stem cells are not dependent on Bcr-Abl kinase activity for their survival. Blood 119: 1501-1510, 2012.

5. Cortes J, Lipton JH, Rea D, et al. Omacetaxine 202 Study Group. Phase 2 study of subcutaneous omacetaxine mepesuccinate after TKI failure in patients with chronic-phase CML with T315I mutation. Blood 120: 2573-2580, 2012.

6. Druker BJ, Talpaz M, Resta DJ, et al. Efficacy and safety of a specific inhibitor of the BCR-ABL tyrosine kinase in chronic myeloid leukemia. N Engl J Med 344: 1031-1037, 2001.

7. Weisberg E, Griffin JD. Resistance to imatinib (Glivec): update on clinical mechanisms. Drug Resist Updat 5: 231-238, 2003.

8. Quintás-Cardama A, Kantarjian HM, Cortes JE. Mechanisms of primary and secondary resistance to imatinib in chronic myeloid leukemia. Cancer Control 16: 122-131, 2009.

9. Nievergall E, Reynolds J, Kok CH, et al. TGF- $\alpha$ and IL-6 plasma levels selectively identify $C M L$ patients who fail to achieve an early molecular response or progress in the first year of therapy. Leukemia 30: 1263-1272, 2016.

10. Nilsson MB, Langley RR, Fidler IJ. Interleukin-6, secreted by human ovarian carcinoma cells, is a potent proangiogenic cytokine. Cancer Res 65: 10794-10800, 2005.

11. Arber DA, Orazi A, Hasserjian R, et al. The 2016 revision to the World Health Organization classification of myeloid neoplasms and acute leukemia. Blood 127: 2391-2405, 2016.

12. Bacigalupo A, Gualandi F, Van Lint MT, et al. Multivariate analysis of risk factors for survival and relapse in chronic granulocytic leukemia following allogeneic marrow transplantation: impact of disease related variables (Sokal score). Bone Marrow Transplant 12: 443-448, 1993. 
International Journal of Hematology and Oncology

13. Hasford J, Pfirrmann M, Hehlmann R, et al. Prognosis and prognostic factors for patients with chronic myeloid leukemia: nontransplant therapy; Collaborative CML Prognostic Factors Project Group. Semin Hematol 40: 4-12, 2003.

14. Hasford J, Baccarani M, Hoffmann V, et al. Predicting complete cytogenetic response and subsequent progression-free survival in 2060 patients with CML on imatinib treatment: the EUTOS score. Blood 118: 686-92, 2011.

15. Hehlmann R, Hocchaus A, Baccarani M. Chronic myeloid leukemia. Lancet 370: 342-350, 2007.

16. Cortes J, Hochhaus A, Hughes T, et al. Front-Line and Salvage Therapies with Tyrosine Kinase Inhibitors and Other Treatment in Chronic Myeloid Leukemia. J Clin Oncol 29: 524-531, 2011.

17. Fabarius A, Leitner A, Hochhaus A, et al. Impact of ACA at diagnosis on prognosis of CML: long-term observation from 1151 patients of the randomized CML study IV. Blood 118: 6760-6768, 2011

18. De Lavallade H, Apperely JF, Khorashad JS, Imatinib for newly diagnosed patients with chronic myeloid leukemia: incidence of sustained responses in an intention-to-treat analysis. J Clin Oncol 26: 3358-3363, 2008.

19. Marin D, Ibrahim AR, Lucas C, et al. Assessment of BCR-ABL transcript levels at 3 months is the only requirement for predicting outcome for patients with chronic myeloid leukemia treated with tyrosine kinase inhibitors. J Clin Oncol 30: 232238, 2012.

20. Nievergall E, Reynolds J, Chung H. et al. Chronic myelogenous leukemia cell line from apoptosis induced by BCR/ABL inhibition. Leukemia 15:1232-1239, 2002.

21. Umemoto $T$, Yamato $M$, Ishihara J. Integrin- $\alpha v B 3$ regulates thrombopoietin-mediated maintenance of hematopoietic stem cells. Blood 119: 83-94, 2012.
22. Reynaud D, Pietras E, Barry-Holson K. IL-6 controls leukemic multipotent progenitor cell fate and contributes to chronic myelogenous leukemia development. Cancer Cell 20: 66173, 2016.

23. Heidel FH, Bullinger L, Feng Z. Genetic and pharmaco-locic inhibition of beta-catenin targets imatinib-resistant leukemia stem cells in CML. Cell Stem Cell 10: 412-24, 2012.

24. Weisberg E, Azab AK, Manley PW, et al. Inhibition of CXCR4 in $\mathrm{CML}$ cells disrupts their interaction with the bone marrow microenvironment and sensitizes them to nilotinib. Leukemia 26: 985-90, 2012

25. Zhang $X$, Tu H, Yang $Y$, et al. High IL-7 levels in the bone marrow microenvironment mediate imatinib resistance and predict disease progression in chronic myeloid leukemia. Int $J$ Hematol 104: 358-367, 2016.

\section{Correspondence}

Mohammed Mansour Abbas EID

Fayoum University, Faculty of Medicine

Department of Clinical Pathology

PO Box 63514, FAYOUM City / EGYPT

Tel: 0021274083431

Fax: 002846302350

e-mail: mma21@fayoum.edu.eg 